\title{
INCIDENTAL DETECTION OF MICROFILARAE IN CYTOLOGICAL SMEARS
}

Parul Joshi' ${ }^{1}$, Shalini Shukla ${ }^{2}$, Jayant Rai ${ }^{3}$, Pankaj Mishra ${ }^{4}$, Ajay Narang55, Umesh paliwal ${ }^{6}$, Neha Ahuja7, Neelima $^{8}$

\section{HOW TO CITE THIS ARTICLE:}

Parul Joshi, Shalini Shukla, Jayant Rai, Pankaj Mishra, Ajay Narang, Umesh paliwal, Neha Ahuja, Neelima. "Incidental Detection of Microfilarae in Cytological Smears". Journal of Evolution of Medical and Dental Sciences 2015; Vol. 4, Issue 82, October 12; Page: 14354-14359, DOI: 10.14260/jemds/2015/2041

ABSTRACT: Filariasis is a major health problem in endemic areas like India. Detection of microfilaria in cytological smear is unusual and association with malignancy is more uncommon. We present ten cases where microfilaria detected incidentally during examination of cytological samples. Eight of them are benign disease entities while two are malignant in nature. CONCLUSION: Microfilaria is public health problem in tropical region of South-East-Asia including Indian subcontinent. Cytology smear can be a very sensitive and cost effective tool for detection of helminthic etiology in unexplained infective pathology and to provide correct treatment.

KEYWORDS: Filariasis, Cytology, Malignancy.

INTRODUCTION: Filariasis is a vector borne disease, is common in India showing inflammatory reaction or can be asymptomatic.[1] Wuchereria bancrofti is found in most of the cases, possibly because of their ability to migrate along with lymphatics. ${ }^{[2]}$ Because of its nocturnal periodicity it is not easy to detect microfilariae in routine peripheral blood smears, cytological smears including body fluids. ${ }^{[3]}$ Microfilariae in malignant pathology is far more rare in cytology. ${ }_{[}^{[4]}$ Here we report ten cases where microfilarae were detected in routine cytology without a clinical suspicion of filariasis. Two of them were associated with malignancy.

CASE 1 Pericardial Fluid: A 58 years old male presented with recurrent pericardial effusion with low grade fever for last 6 months. The aspirates was hemorrhagic. Cytological smears showed presence of microfilaria along with lymphocytes in the background of RBCs.

CASE 2 Axillary Swelling: A 30 years old female presented with axillary swelling since 1 month. There was no other significant complaint. Cytological smears showed presence of numerous microfilaria along with lymphocytes and few macrophages in the background of proteinaceous material and degenerated cells.

CASE 3 Cervical Swelling: A 60 years old male presented with right cervical swelling since 3 month. There was history of tobacco chewing since 40 years, significant weight loss and loss of appetite. Aspirate was blood mixed. Cytological smears showed presence of microfilaria along with atypical malignant looking cells in sheets, clusters, forming acini as well as scattered singly in the background of fibrinous material.

CASE 4 Pleural Fluid: A 32 years male presented with pleural effusion with chest pain and fever since 2 month. The aspirate was hemorrhagic. Cytological smears showed presence of microfilaria along with lymphocytes and polymorphs in the background of RBCs. 


\section{ORIGINAL ARTICLE}

CASE 5 Pap Smear: A 44 years old female presented with foul smelling discharge per vaginum since 4 month. Pap smear showed numerous microfilaria with adherent inflammatory cells and epithelial cells along with squamous metaplasia with reactive atypia.

CASE 6 Cervical Swelling: A 25 years old male presented with cervical swelling since 1 month. There was no other significant complaint. Cytological smears showed presence of microfilaria along with polymorphous population of lymphoid series.

CASE 7 Pap Smear: A 33 years old female presented with post coital bleeding and pain in lower abdomen since 3 months. Pap smear showed numerous microfilaria with acute and chronic inflammatory cells and epithelial cells.

CASE 8 Ascitic Fluid: A 38 years old female presented with recurrent ascitis, fever and weakness since 7 months. The aspirate was clear. Cytological smears showed presence of microfilariae admixed with lymphocytes along with reactive mesothelial cells.

CASE 9 Cervical Swelling: A 55 years old male presented with left cervical swelling since 3 months with pain and rapid increase in size from last 15 days. Cytological smears show few malignant highly pleomorphic cells along with numerous acute, chronic inflammatory infiltrate. Few microfilaria were noted.

CASE 10 Testis: 32 years old male presented with fever, pain in testicular area since one month. On aspiration blood mixed aspirate was obtained. Smears revealed moderate cellularity with polymorphonuclear cells, degenerated macrophages. Focal areas show numerous microfilariae.

Fig. 1: Microfilaria of $\mathrm{W}$. bancrofti along with inflammatory cells in fine needle aspirate of lymph node (Hematoxylin- Eosin, X40).

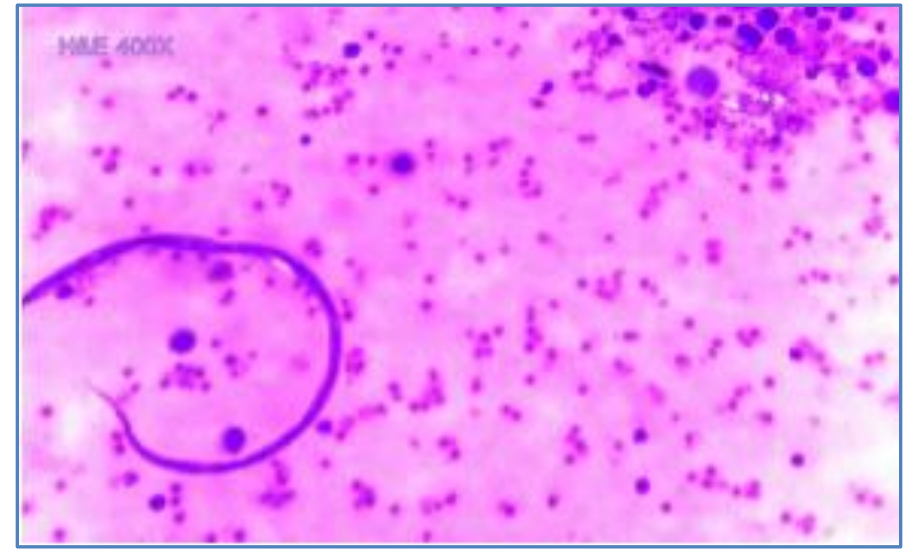

Fig. 1 


\section{ORIGINAL ARTICLE}

Fig. 2: Microfilaria of W. bancrofti along with inflammatory cells in fine needle aspirate of testis (Hematoxylin- Eosin, X 40).

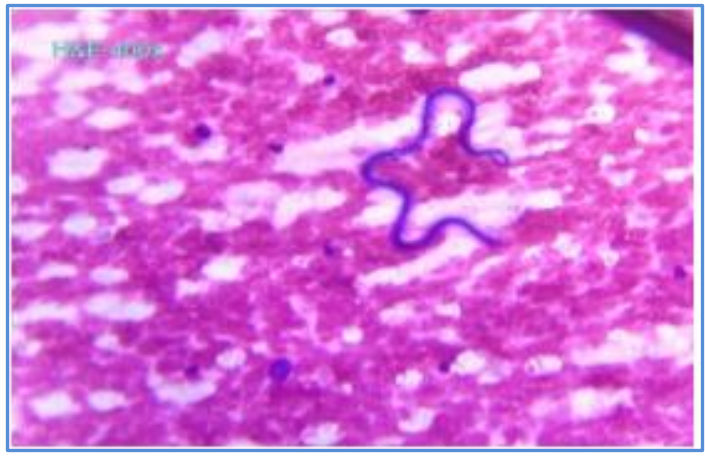

Fig. 2

Fig. 3: Microfilaria of $\mathrm{W}$. bancrofti along with benign squamous cells and inflammatory cells in pap smear (Hematoxylin- Eosin, X 40)

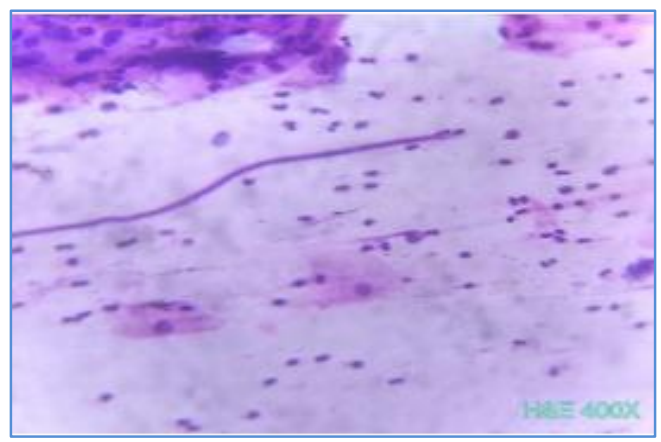

\section{Fig. 3}

Fig. 4: Microfilaria of W. bancrofti along with lymphoid cells in fluid cytology (Hematoxylin-Eosin, $\mathrm{X} 40$ ).

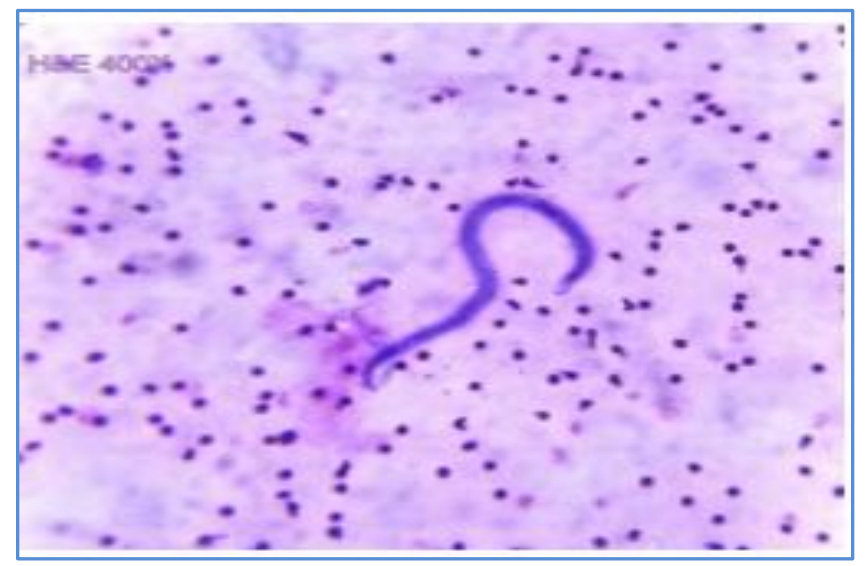

Fig. 4 


\section{ORIGINAL ARTICLE}

Fig. 5: Microfilaria of W. bancrofti along with inflammatory cells in fine needle aspirate of lymph node (Hematoxylin- Eosin, X 40).

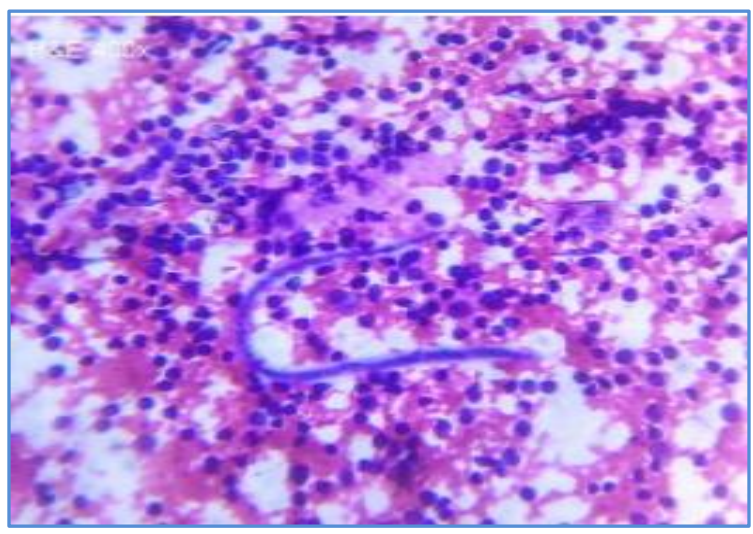

Fig. 5

DISCUSSION: Microfilaria is a major health problem of South-East-Asia including Indian subcontinent. It is caused by the nematode W. bancrofti, Brugia malayi, Brugia tumori and transmitted through bite of Culex mosquitoes.[2] The microfilaria of W. bancrofti is differentiated from the microfilariae of B. malayi by its bigger size, straightened margins without secondary kinks, prominent somatic nuclei, nuclei free tail tip on morphology. ${ }^{[3]}$ Adult parasite live in lymph vessels and many times causes acute, chronic inflammatory reaction and obstruction leading to lymphoedema, lymphangiovarix.[4] fibrosis. Some infected individuals remain asymptomatic. They are traditionally called as "endemic normals." [5]

They were seen occasionally during routine cytological and histological examination from various benign lesions and they have rarely been detected in association with neoplastic lesions in cytological smears. In most of the reported cases microfilaria have been detected in lymph node, bone marrow, pleural and pericardial fluid, ovarian cyst fluid. .6] These parasite has been detected in bone marrow, lymph node aspirates, most being benign lesions with only few malignant cases.[6,7,8]

Most of the authors explained that microfilaria circulate in the vasculature, lymphatic channels and whenever the neoplastic lesion causes obstruction of these channels they appear in the tissue fluid or come to the surface material. In neoplastic lesion increased blood vasculature also causes the increase deposition of microfilaria to these sites.[9] Ahluwalia et al., stated that larva may be present in the vasculature and aspiration may lead to the rupture of vessel and release of microfilaria into the aspirate from neoplasm.[10] Gupta et al. reported six cases where microfilaria were found in body fluids cytology and FNAC smears in association with tubercular pleural effusion/lymphadenitis, pregnancy, non-Hodgkin's lymphoma, malnutrition and young age. Incidental detection of microfilaria in a routine pap smear in absence of blood parasitemia in very as we have reported in this article.

In our view careful screening of cytological smears should be done for detection of coexistent microfilarial infestation with benign or malignant conditions so as to reduce parasitic burden and to provide accurate treatment. 
CONCLUSION: The association of microfilariasis should be kept in mind in endemic areas like India. FNAC smears can be very sensitive and cost effective tool for the detection of helminthic etiology, especially in malignant cases where we find unexplained acute, chronic inflammatory infiltrate.

To summarise filariasis may be detected in clinically unsuspected cases, so high index if suspicious should be kept in mind and careful screening is mandatory for the search of coexisting pathology.

\section{REFERENCES:}

1. Katti TV, Athanikar VS, Ananthrao AS, Rathod CV, Cytodiagnosis of microfilarial lymphadenitis coexistent with metastatic squamous cell carcinoma in a left cervical lymph node: An unusual presentation. Ann Nigerian Med.2012; 6: 47-49.

2. Park K. Park's Textbook of Preventive and Social Medicine. 18th ed. Jabalpur, India: Bhanot Publishers; 2005.

3. Gangopadhyay M, Biswas B, Chowdhury M, Deoghoria D. Microfilaria in thyroid aspirate- An unexpected finding. J Cytol. 2011; 28: 240-241.

4. Varghese R, Raghuveer CV, Pai MR, Bansal R. Microfilariae in cytologic smears: A report of six cases. Acta Cytol. 1996; 40:299-301.

5. Jha A, Shrestha R, Aryal G, Pai MR, Bansal R. Microfilarae in cytologic smears: a report of six cases. Acta Cytol.1996; 40:29-301.

6. Zafar U, Rahman K, Sherwani RK, Shahid M. Microfilalariae of Wucheria bancrofti in bone marrow. Indian J Hematol Blood Transfus. 2009; 25:42-3.

7. Yenkeshwar PN, Kumbhalkar DT, Bobhate SK. Microfilariae in fine needle aspirates: A report of 22 cases .Indian J Pathol Microbiol. 2006; 49:365-9.

8. Kolte SS, Satarkar RN, Mane PM. Microfilaria concomitant with metastatic deposits of adenocarcinoma in lymph node fine needle aspiration cytology: A chance finding. J Cytol. 2010; 27:78-80.

9. Gupta S, Sodhani P, Jain S, Kumar N. Microfilariae in association with neoplastic lesions: Report of five cases. Cytopathology. 2001; 12:120-6.

10. Ahluwalia C, Choudhary M, Bajaj P. Incidental detection of microfilariae in aspirates from Ewing's sarcoma of bone. Diagn Cytopathol. 2003; 29:31-2. 


\section{ORIGINAL ARTICLE}

\section{AUTHORS:}

1. Parul Joshi

2. Shalini Shukla

3. Jayant Rai

4. Pankaj Mishra

5. Ajay Narang

6. Umesh paliwal

7. Neha Ahuja

8. Neelima

\section{PARTICULARS OF CONTRIBUTORS:}

1. Assistant Professor, Department of Pathology, Rama Medical College.

2. Consultant Pathologist, Department of Pathology, Paliwal Diagnostics.

3. Junior Resident, Department of Pharmacology, GMC, Surat.

4. Consultant Pathologist, Department of Pathology, Paliwal Diagnostics.

\section{FINANCIAL OR OTHER}

COMPETING INTERESTS: None
5. Associate Professor, Department of Pathology, Rama Medical College.

6. Associate Professor, Department of Pathology, Rama Medical College

7. Consultant Pathologist, Department of Pathology, Paliwal Diagnostics

8. Consultant Pathologist, Department of Pathology, Paliwal Diagnostics.

\section{NAME ADDRESS EMAIL ID OF THE CORRESPONDING AUTHOR:}

Dr. Parul Joshi,

Paliwal Diagnostics, 117/H - 1/02,

Pandu Nagar, Kanpur-05.

E-mail: yamini.joshi4@gmail.com, drshalinishukla.8@gmail.com.

Date of Submission: 17/09/2015.

Date of Peer Review: 21/09/2015.

Date of Acceptance: 01/10/2015.

Date of Publishing: 10/10/2015. 